\title{
Viscous Critical-Layer Analysis of Vortex Normal Modes
}

\author{
By Stéphane Le Dizès
}

The linear stability properties of an incompressible axisymmetrical vortex of axial velocity $W_{0}(r)$ and angular velocity $\Omega_{0}(r)$ are considered in the limit of large Reynolds number. Inviscid approximations and viscous WKBJ approximations for three-dimensional linear normal modes are first constructed. They are then shown to be singular at the critical points $r_{c}$ satisfying $\omega=$ $m \Omega_{0}\left(r_{c}\right)+k W_{0}\left(r_{c}\right)$, where $\omega$ is the frequency, $k$ and $m$ the axial and azimuthal wavenumbers. The goal of this paper is to resolve these singularities. We show that a viscous critical-layer analysis is analytically tractable. It leads to a single sixth-order equation for the perturbation pressure. This equation is identical to the one obtained in stratified shear flows for a Prandtl number equal to 1. Integral expressions for typical solutions of this equation are provided and matched to the outer inviscid and viscous approximations in the complex plane around $r_{c}$. As for planar flows, it is proved that the critical layer solution with a balanced behavior matches a non-viscous approximation in a $4 \pi / 3$ sector of the complex-plane. As a consequence, the Frobenius expansions of a non-viscous mode on each side of a critical point $r_{c}$ differ by a $\pi$ phase jump.

\footnotetext{
Address for correspondence: Stéphane Le Dizès, Institut de Recherche sur les Phénomènes Hors Équilibre, 49, rue F. Joliot-Curie, B.P. 146, F-13384 Marseille cedex 13, France: e-mail: ledizes@irphe.univ-mrs.fr. 


\section{Introduction}

The stability of rotating flows with or without axial flow is an important issue in numerous situations ranging from engineering to geophysics. Moreover, applications are often concerned with the large Reynolds number limit, which constitutes a singular limit in the linear stability problem. For planar shear flows, such a limit has been the subject of a considerable amount of work, and several textbooks that elucidate the theory are now available [1-3]. For rotating flows, the asymptotic theory of the stability problem is, by contrast, much less advanced. The goal of this paper is to provide a first step in formulating an asymptotic theory. More specifically, our purpose is to resolve the simple singularities (critical points, turning points) that inviscid and viscous approximations of linear modes exhibit in the large Reynolds number limit.

The importance of critical points in the hydrodynamic stability theory of shear flows is now well understood (see, for instance, the text books by Lin [1] or Drazin and Reid [2]). For two-dimensional modes in planar shear flows, these singular points occur where the phase velocity of the perturbation equals the basic flow velocity. As first shown by Lin, these singularities can be smoothed out in a so-called viscous critical layer of width $O\left(R e^{-1 / 3}\right)$ around the critical point. The viscous critical layer equation is a fourth-order equation of Airy type. The behavior of its solutions for large complex values of its argument provides the nature of the solution around the critical point. In particular, Lin [4] demonstrated that the presence of a critical point implies the existence of large regions of the complex plane where viscosity is always important however large the Reynolds number. Numerous works have been published on this issue and we refer to the book by Drazin and Reid [2] for details and references. More complex critical point singularities exist in planar flows when additional effects are present. For a stratified flow, the critical layer equation is of sixth order and its resolution involves a more sophisticated analysis [5,6]. Discussions and references on other aspects of critical layers in parallel shear flows can be found in Maslowe [7], Cowley and $\mathrm{Wu}$ [8], and Churilov and Shukhman [9].

One of the difficulties of the stability theory for rotating flows is that viscous perturbation equations do not reduce to a simple single equation such as the Orr-Sommerfeld equation for planar flows. Three-dimensional viscous instability modes are governed by a complicated system of at least two coupled equations that cannot be simplified. In particular, there does not exist any "Squire theorem" for swirling flows, which permits one to reduce the normal mode analysis to two-dimensional modes. Most results have thus been obtained by numerical analysis. The reader is referred to the review of Ash and Khorrami [10] for a comprehensive discussion of available results in the literature.

Critical point singularities are known to occur in the non-viscous stability problem for a swirling flow [see, for instance, 11, 13]. So far, these singularities have been avoided by integrating the stability equations in the complex 
plane by assuming that the inviscid approximation can be continued around the critical point as for planar flows [4]. The present paper will provide a justification of this assumption. Moreover, it will also provide approximations for the solution in the regions where the behavior of the solution is dominated by viscosity. More specifically, the critical point singularity will be resolved by a viscous critical layer analysis. A simple equation governing the spatial structure of the three-dimensional normal mode pressure in the viscous critical layer will be derived. Surprisingly, this equation will turn out to be identical to the critical layer equation obtained for the perturbation transverse velocity in a stratified shear flow with a unit Prandtl number. This equation was first solved by Koppel [5] using integral expressions for the solutions. Koppel [5] also provided the asymptotic behavior of typical solutions. His results were extended and used to obtain the dispersion relation of particular flows [14-16]. Interestingly, Hughes and Reid [14] also obtained the same critical-layer equation for the axisymmetric modes in the flow between rotating cylinders in the narrow gap approximation. It is surprising that nobody has tried to use this strong indication to show that the same critical-layer equation is also valid for general rotating flows.

As demonstrated by Koppel [5], the critical-layer equation possesses solutions with a balanced behavior and with a dominant-recessive behavior as for the classical critical layer equation. The same type of solutions, based on definitions given in [14] and [17] will be used here. They will also be matched to outer viscous and inviscid solutions in the full complex neighborhood of the critical point.

This paper is organized as follows. Viscous and inviscid approximations for the normal modes are first derived in section 2. Both are shown to be singular at the critical points. The viscous critical layer equation is derived and integrated in section 3 . The matching between critical layer solutions and outer approximations are performed in section 4 . The last section briefly summarizes the results.

\section{Three-dimensional linear normal modes}

\subsection{Basic flow and perturbation equations}

Consider a general rotating (swirling) flow with axial flow whose velocity field in cylindrical coordinates is of the form

$$
\mathbf{U}_{\mathbf{0}}(r)=\left(0, V_{0}(r), W_{0}(r)\right) .
$$

Both the azimuthal velocity $V_{0}(r)$ and the axial velocity $W_{0}(r)$ depend on the radial coordinate $r$ only. It is also useful to define from $V_{0}(r)$, the angular 
velocity $\Omega_{0}(r)$ and the axial vorticity $\omega_{0}(r)$ :

$$
\Omega_{0}(r)=\frac{V_{0}(r)}{r}
$$

and

$$
\omega_{0}(r)=\frac{1}{r} \frac{d\left(r V_{0}\right)}{d r} .
$$

Such a basic flow is, in general, not a solution of the Navier-Stokes equations in the presence of viscosity. Both the axial vorticity and the axial velocity satisfy a diffusion equation, which means that profiles vary slightly in time for large Reynolds numbers. As is commonly done in stability analysis, this viscous diffusion is neglected in the present study.

Linear perturbations can then be expressed in the form of normal modes

$$
(\mathbf{U}, P)=(u, v, w, p) e^{i k z+i m \theta-i \omega t},
$$

where $k$ and $m$ are axial and azimuthal wavenumbers and $\omega$ is the frequency. The velocity and pressure amplitudes $(u, v, w, p)$ then satisfy the linear system:

$$
\begin{gathered}
\Phi u-2 \Omega_{0} v=-\frac{\partial p}{\partial r}+\frac{1}{R e}\left(\Delta u-\frac{u}{r^{2}}+\frac{2 i m v}{r^{2}}\right) \\
\Phi v+\omega_{0} u=-\frac{i m p}{r}+\frac{1}{R e}\left(\Delta v-\frac{v}{r^{2}}-\frac{2 i m u}{r^{2}}\right) \\
\Phi w+W_{0}^{\prime} w=-i k p+\frac{1}{R e} \Delta w \\
\frac{1}{r} \frac{\partial(r u)}{\partial r}+\frac{i m v}{r}+i k w=0,
\end{gathered}
$$

where a prime denotes a derivative with respect to $r, \Delta=\frac{\partial^{2}}{\partial r^{2}}+\frac{1}{r} \frac{\partial}{\partial r}-\frac{m^{2}}{r^{2}}$, and

$$
\Phi(r)=-i \omega+i m \Omega_{0}(r)+i k W_{0}(r) .
$$

The Reynolds number $R e$ is here constructed using characteristic scales of the basic flow such as the vortex core size and the maximum axial velocity, or the maximum angular velocity. In the following, we assume only that the Reynolds number is large to construct an asymptotic theory with respect to the small parameter $1 / R e$.

For large Reynolds numbers, approximate solutions can be sought by expanding the function in powers of $1 / R e$. If the terms proportional to $1 / R e$ in the system (5) are negligible, the leading order solution can be considered as inviscid. Approximations for these non-viscous solutions are considered in the next section. 
The terms proportional to $1 /$ Re may also be non-negligible at leading order. This occurs when the perturbation varies on a fast (viscous) scale $r_{v}=\sqrt{\operatorname{Re}}$. Approximations for these "viscous" solutions are constructed in section 2.3. Both viscous and inviscid approximations will turn out to be singular at the radial coordinates $r_{c}$, where $\Phi\left(r_{c}\right)=0$. These points are called critical points and satisfy

$$
\omega=m \Omega_{0}\left(r_{c}\right)+k W_{0}\left(r_{c}\right)
$$

The goal of this paper will be to resolve these singularities to provide the matching condition of viscous and inviscid approximations across and around critical points. The regular singularity at $r=0$ is not considered. In particular, it is assumed that the critical point is not at the origin.

\subsection{Inviscid approximation}

As defined above, the non-viscous approximation is the leading order approximation of a viscous solution for which the viscous terms in (5) (i.e., the terms multiplied by $1 / R e$ ) are negligible. If we are far from the critical points and from the origin, an inviscid approximation can be sought as an expansion in powers of $1 / \operatorname{Re}$ as

$$
\begin{aligned}
& u(r, R e)=u_{0}(r)+\frac{1}{R e} u_{1}(r)+\cdots \\
& v(r, R e)=v_{0}(r)+\frac{1}{R e} v_{1}(r)+\cdots \\
& w(r, R e)=w_{0}(r)+\frac{1}{R e} w_{1}(r)+\cdots \\
& p(r, R e)=p_{0}(r)+\frac{1}{R e} p_{1}(r)+\cdots
\end{aligned}
$$

System (5) then becomes at leading order

$$
\begin{aligned}
\Phi u_{0}-2 \Omega_{0} v_{0} & =-\frac{d p_{0}}{d r} \\
\Phi v_{0}+\omega_{0} u_{0} & =-\frac{i m p_{0}}{r} \\
\Phi w_{0}+W_{0}^{\prime} u_{0} & =-i k p_{0} \\
\frac{1}{r} \frac{d\left(r u_{0}\right)}{d r}+\frac{i m v_{0}}{r}+i k w_{0} & =0 .
\end{aligned}
$$

This system can be reduced to a single equation for the pressure $p_{0}$, namely, 


$$
\begin{aligned}
& \frac{d^{2} p_{0}}{d r^{2}}+\left(\frac{1}{r}-\frac{\Delta_{0}^{\prime}}{\Delta_{0}}\right) \frac{d p_{0}}{d r} \\
& +\left(\frac{2 m i}{r \Phi \Delta_{0}}\left(\Omega_{0}^{\prime} \Delta_{0}-\Omega_{0} \Delta_{0}^{\prime}\right)-\frac{k^{2} \Delta_{0}}{\Phi^{2}}-\frac{m^{2}}{r^{2}}+\frac{2 m k W_{0}^{\prime} \Omega_{0}}{r \Phi^{2}}\right) p_{0}=0,
\end{aligned}
$$

where $\Delta_{0}(r)=2 \omega_{0}(r) \Omega_{0}(r)+\Phi^{2}(r)$. This equation is given in Saffman's book [18], page 244, in the configuration without axial flow.

The simple zeroes of $\Phi(r)$ are regular singularities of equation (10). Near such critical points $r_{c}, p_{0}$ behaves as

$$
p_{0} \sim\left(r-r_{c}\right)^{\alpha}
$$

where

$$
\alpha(\alpha-1)=K_{c}
$$

with

$$
K_{c}=\frac{2 \Omega_{0 c} k\left(k \omega_{0 c}-m W_{0 c}^{\prime} / r_{c}\right)}{\left(\Phi_{c}^{\prime}\right)^{2}}=-\frac{2 \Omega_{0 c} k\left(k \omega_{0 c}-m W_{0 c}^{\prime} / r_{c}\right)}{\left(m \Omega_{0 c}^{\prime}+k W_{0 c}^{\prime}\right)^{2}} .
$$

The subscript $c$ in the above expression indicates values taken at $r_{c}$. To each root $\alpha^{(a)}$ and $\alpha^{(b)}$ of (12) corresponds a leading order pressure $p_{0 n v}^{(a)}$ and $p_{0 n v}^{(b)}$ with the behavior (11). We denote $p_{n v}^{(a)}$ and $p_{n v}^{(b)}$ the non-viscous approximation (8d) constructed with these leading-order expressions. The coefficients $\alpha^{(a)}$ and $\alpha^{(b)}$ are a priori both complex. This means that $r_{c}$ is a branch point singularity of both inviscid solutions $p_{n v}^{(a)}$ and $p_{n v}^{(b)}$. This implies that none of the inviscid solutions can be uniformly valid around $r_{c}$ in the complex r-plane. The resolution of the singularity, which will be performed in section 3, will determine how the non-viscous solution can be continued in the complex plane.

\subsection{Viscous WKBJ approximation}

Viscous solutions are the solutions for which the viscous term in system (5d) is present at leading order. A WKBJ approximation for such a solution can be sought in the form

$$
\begin{aligned}
& u(r, R e)=\frac{1}{\sqrt{R e}}\left(u_{1}(r)+\frac{1}{\sqrt{R e}} u_{2}(r)+\cdots\right) e^{\sqrt{R e} \phi(r)}, \\
& v(r, R e)=\left(v_{0}(r)+\frac{1}{\sqrt{R e}} v_{1}(r)+\cdots\right) e^{\sqrt{R e} \phi(r)},
\end{aligned}
$$




$$
\begin{aligned}
& w(r, R e)=\left(w_{0}(r)+\frac{1}{\sqrt{\operatorname{Re}}} w_{1}(r)+\cdots\right) e^{\sqrt{\operatorname{Re}} \phi(r)}, \\
& p(r, \operatorname{Re})=\frac{1}{\sqrt{\operatorname{Re}}}\left(p_{1}(r)+\frac{1}{\sqrt{\operatorname{Re}}} p_{2}(r)+\cdots\right) e^{\sqrt{\operatorname{Re}} \phi(r)} .
\end{aligned}
$$

This gives, at leading order, $\left[\phi^{\prime}(r)\right]^{2}=\Phi(r)$, i.e.,

$$
\phi^{ \pm}(r)= \pm \int_{r_{c}}^{r} \sqrt{\Phi(s)} d s
$$

where $r_{c}$ has been chosen in the integral to simplify the matching procedure done in section 4.

At the second order, as for the non-viscous equations, an equation can be obtained for the pressure amplitude alone. This equation reads, for both phases $\phi^{+}$and $\phi^{-}$,

$$
\left(2 \Phi \frac{d}{d r}+i k W_{0}^{\prime}\right) \frac{1}{\omega_{0}}\left(2 \Phi \frac{d}{d r}+i k \Omega_{0}^{\prime}\right) q_{1}=k\left(2 k \Omega_{0}-\frac{m W_{0}^{\prime}}{r}\right) q_{1},
$$

where $q_{1}$ is related to $p_{1}$ by

$$
q_{1}=\frac{(\Phi)^{3 / 4} \sqrt{r}}{\Omega_{0}} p_{1}
$$

For each phase $\phi^{+}$and $\phi^{-}$, there exists two independent solutions of this equation. This means that there are four independent viscous solutions. It is easy to show that $p_{1}$ is singular at points where $\Phi=0$. These points are in fact turning points of the WKBJ approximation [19]. Near such points $r_{c}, p_{1}$ behave as

$$
p_{1} \sim\left(r-r_{c}\right)^{\beta}
$$

where $\beta$ satisfies

$$
4 \beta^{2}+8 \beta+15 / 4=K_{c}
$$

with $K_{c}$ defined by (13).

We denote $p_{1 v}^{(a)}$ and $p_{1 v}^{(b)}$, the two independent solutions which satisfy (18), with $\beta=\beta^{(a)}$ and $\beta=\beta^{(b)}$, respectively. The four viscous approximations (14d) constructed with these leading-order solutions and with each phase $\phi^{+}$ and $\phi^{-}$will be denoted by $p_{v}^{(a)+}, p_{v}^{(a)-}, p_{v}^{(b)+}$, and $p_{v}^{(b)-}$. As for the non-viscous solutions, because both roots $\beta^{(a)}$ and $\beta^{(b)}$ of (19) are a priori complex, $r_{c}$ is again a branch point singularity for each viscous approximation. None of these approximations is therefore uniformly valid around $r_{c}$ in the complex $r$-plane. The critical layer analysis, performed in the next section, will provide the domains of uniform validity. 


\section{Critical layer analysis}

Both the turning point singularity of the viscous approximation and the critical point singularity of the inviscid approximation can be smoothed out in a viscous critical layer. The size of the critical layer is, as for planar shear flows, $O\left(R e^{-1 / 3}\right)$. Following classical asymptotic matching analysis, we therefore introduce the local variable

$$
\bar{r}=R e^{1 / 3}\left(r-r_{c}\right) .
$$

The local solution is sought in powers of $R^{-1 / 3}$ :

$$
\begin{aligned}
\bar{u}(\bar{r}, R e) & =\operatorname{Re}^{-1 / 3}\left(\bar{u}_{1}(\bar{r})+\operatorname{Re}^{-1 / 3} \bar{u}_{2}(\bar{r})+\cdots\right), \\
\bar{v}(\bar{r}, R e) & =\left(\bar{v}_{0}(\bar{r})+\operatorname{Re}^{-1 / 3} \bar{v}_{1}(\bar{r})+\cdots\right), \\
\bar{w}(\bar{r}, R e) & =\left(\bar{w}_{0}(\bar{r})+\operatorname{Re}^{-1 / 3} \bar{w}_{1}(\bar{r})+\cdots\right), \\
\bar{p}(\bar{r}, R e) & =\operatorname{Re}^{-1 / 3}\left(\bar{p}_{1}(\bar{r})+\operatorname{Re}^{-1 / 3} \bar{p}_{2}(\bar{r})+\cdots\right),
\end{aligned}
$$

which, when substituted in (5), yields the leading-order equations

$$
\begin{aligned}
-2 \Omega_{0 c} \bar{v}_{0}= & -\frac{d \bar{p}_{1}}{d \bar{r}}, \\
\omega_{0 c} \bar{u}_{1}= & -\frac{i m \bar{p}_{1}}{r_{c}}+\frac{d^{2} \bar{v}_{0}}{d \bar{r}^{2}}-\Phi_{c}^{\prime} \bar{r} \bar{v}_{0}, \\
W_{0 c}^{\prime} \bar{u}_{1}= & -i k \bar{p}_{1}+\frac{d^{2} \bar{w}_{0}}{d \bar{r}^{2}}-\Phi_{c}^{\prime} \bar{r} \bar{w}_{0}, \\
& \frac{d \bar{u}_{1}}{d \bar{r}}+\frac{i m \bar{v}_{0}}{r_{c}}+i k \bar{w}_{0}=0 .
\end{aligned}
$$

As before, the subscript $c$ in these equations indicates values taken at $r_{c}$.

A single equation can be obtained for the pressure $\bar{p}_{1}$ by manipulating the above equations, namely,

$$
\left(\frac{d^{2}}{d \bar{r}^{2}}-\Phi_{c}^{\prime} \bar{r}\right)\left(\frac{d^{2}}{d \bar{r}^{2}}-\Phi_{c}^{\prime} \bar{r}\right) \frac{d^{2} \bar{p}_{1}}{d \bar{r}^{2}}=H_{c} \bar{p}_{1},
$$

with $H_{c}=2 \Omega_{0 c} k\left(k \omega_{0 c}-m W_{0 c}^{\prime} / r_{c}\right)$. If we define

$$
s=\left(\Phi_{c}^{\prime}\right)^{1 / 3} \bar{r}
$$

we obtain

$$
\left(\frac{d^{2}}{d s^{2}}-s\right)\left(\frac{d^{2}}{d s^{2}}-s\right) \frac{d^{2} \bar{p}_{1}}{d s^{2}}=K_{c} \bar{p}_{1},
$$

where $K_{c}$ is as defined in (13). 
This equation is identical to the viscous critical-layer equation obtained in stratified flow for a unit Prandtl number [5]. As shown by Koppel [5], it admits solutions of the form

$$
\bar{p}_{1}(s)=\int_{\mathcal{L}} z^{\gamma} \exp \left(-z^{3} / 3+s z\right) d z,
$$

where $\gamma$ is a complex number satisfying

$$
(\gamma+2)(\gamma+1)=K_{c} .
$$

Consistent with the notation for $\alpha$ and $\beta$ in the previous section, we shall denote $\gamma^{(a)}$ and $\gamma^{(b)}$ the two roots of (27). The contour $\mathcal{L}$ in (26) is a contour in the complex z-plane, which ends at infinity in one of the steepest directions of the phase of the exponential factor, i.e.,

$$
\arg (z) \sim\{0,2 \pi / 3,4 \pi / 3\} \quad \text { as } \quad|z| \rightarrow \infty \text { and } \mathrm{z} \in \mathcal{L} .
$$

The choice of the contour $\mathcal{L}$, and the position of the branch cut issuing from 0 , govern the nature of the solution and its behavior for large $|s|$ in the complex s-plane.

In the following we consider two typical solutions that exhibit a dominantrecessive behavior and a balanced behavior. These solutions correspond to the integration contours $\mathcal{D}_{1}$ and $\mathcal{C}_{1}$ shown in Figure 1 with a branch cut along the positive real axis. They will be denoted by $A_{1}$ and $B_{1}$, respectively:

$$
\begin{aligned}
& A_{1}(s, \gamma)=\frac{1}{2 \pi i} \int_{\mathcal{D}_{1}} z^{\gamma} \exp \left(-z^{3} / 3+s z\right) d z, \\
& B_{1}(s, \gamma)=\frac{1}{2 \pi i} \int_{\mathcal{C}_{1}} z^{\gamma} \exp \left(-z^{3} / 3+s z\right) d z .
\end{aligned}
$$

In Koppel, these solutions correspond to $w_{2}(\eta)$ and $w_{4}(\eta)$. The notation for $A_{1}$ is in agreement with the notation of Reid [17]. It differs from [14] by a constant factor. Note, however, that because Reid only considered integer values of $\gamma$, he did not have to define any branch cut. The position of the branch cut is here an important parameter as it influences the behavior of the solution for large $|s|$. The definition of the solution $B_{1}(s, \gamma)$ is close to the one given by [14] for real $\gamma$ satisfying $\gamma<-1$. For $\gamma>-1, B_{1}(s, \gamma)$ was defined differently with $\mathcal{L}=(0,+\infty)$ in $(26)$. This definition is less general because it requires $\Re e(\gamma)>$ -1 . We therefore prefer our definition (which was also the one used by Koppel) which applies for all complex $\gamma$ except for positive integers.

As is the case for the generalized Airy functions of Reid [see the appendix of 2], other solutions can be obtained by rotating in the complex z-plane the contours $\mathcal{D}_{1}$ and $\mathcal{C}_{1}$ and the branch cut by angles $2 \pi / 3$ and $4 \pi / 3$ around the 


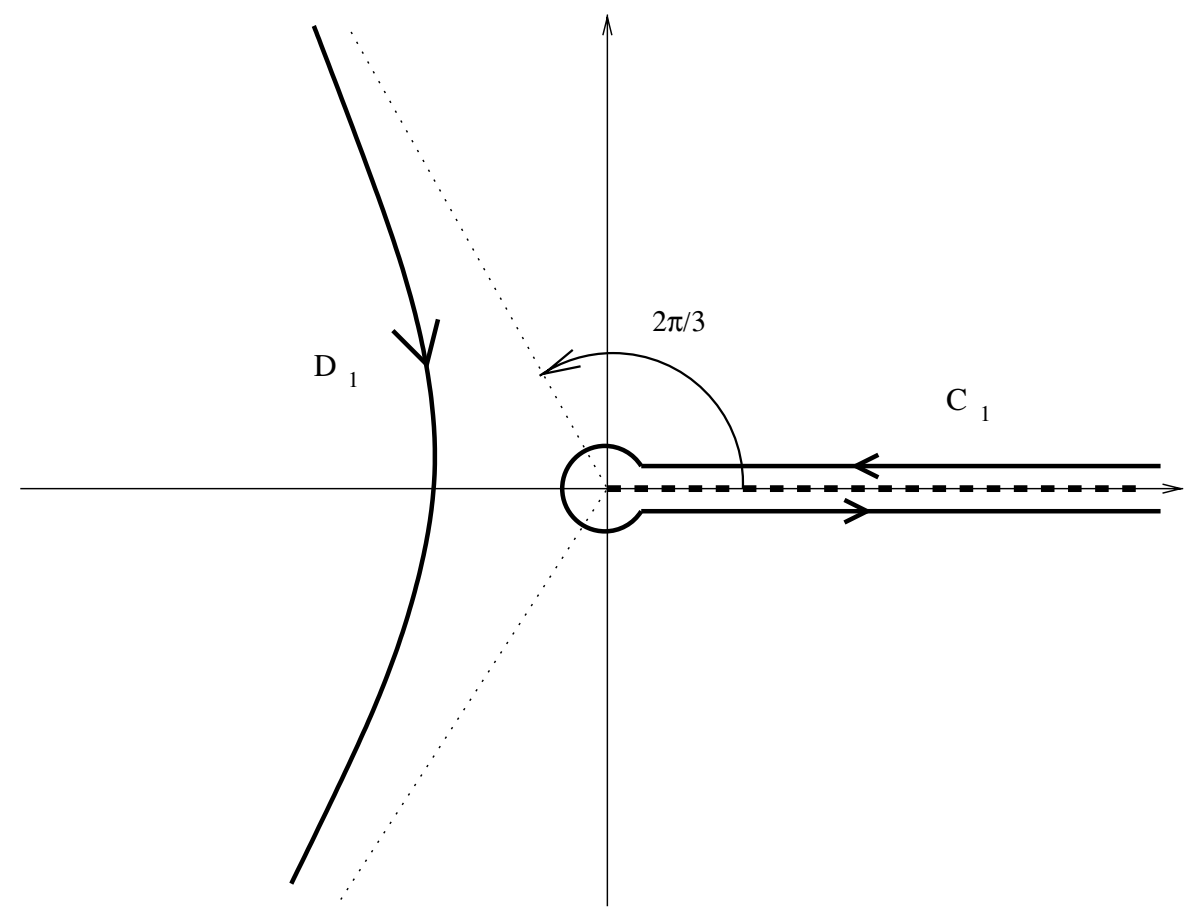

Figure 1. Integration contours $\mathcal{C}_{1}$ and $\mathcal{D}_{1}$ in the complex z-plane. The branch cut is indicated by the dashed line.

origin. These solutions, denoted $A_{2}, A_{3}$ and $B_{2}, B_{3}$ are related to $A_{1}$ and $B_{1}$ by rotation formulas of the form

$$
A_{2}(s, \gamma)=e^{2 i \pi(\gamma+1) / 3} A_{1}\left(s e^{2 i \pi / 3}, \gamma\right),
$$

and

$$
A_{3}(s, \gamma)=e^{4 i \pi(\gamma+1) / 3} A_{1}\left(s e^{4 i \pi / 3}, \gamma\right) .
$$

Similar formulas exist for $B_{2}$ and $B_{3}$ in terms of $B_{1}$.

The asymptotic properties of the functions $A_{k}(s, \gamma)$ and $B_{k}(s, \gamma)$ have been studied in several previous works $[5,6,14,17]$. Thus, only the main steps of the analysis are given here. The asymptotic behaviors of the functions $A_{1}$ and $B_{1}$ for large $|s|$ can be obtained by the steepest descent method as shown in several textbooks $[20,21]$. To apply the method, expression (26) is first transformed by the change of variable $y=\sqrt{|s|} z$ into

$$
p(s, \gamma)=|s|^{(\gamma+1) / 2} \int_{\mathcal{L}^{\prime}} y^{\gamma} \exp \left(-|s|^{3 / 2} \psi_{\theta}(y)\right) d y,
$$


where

$$
\psi_{\theta}(y)=\frac{y^{3}}{3}-e^{i \theta} y
$$

and

$$
\theta=\arg (s)
$$

One then looks at the level contours of the real and imaginary parts of the phase $\psi_{\theta}(y)$ and deforms the integration contour (as well as the branch cut) such that it follows the steepest descent paths of $\Re e\left(\psi_{\theta}\right)$. The contributions for large $|s|$ to the integral come from the saddle points of the phase $\left(\psi_{\theta}^{\prime}(y)=0\right)$ or from the branch points which cannot be removed. Here there are two saddle points $y_{s}^{ \pm}= \pm \exp (i \theta / 2)$ and the branch point 0 . The contribution from 0 is given by

$$
\bar{p}^{(0)}(s, \gamma) \sim e^{2 \pi i \gamma} \sum_{k=0}^{\infty} \frac{s^{-\gamma-1-3 k}(-1)^{k}}{3^{k} k ! \Gamma(-\gamma-3 k)}, \quad \text { as } \quad|\mathrm{s}| \rightarrow \infty,
$$

while the saddle-point contributions are of the form

$\bar{p}^{ \pm}(s, \gamma) \sim \frac{1}{2 \sqrt{\pi} i} \exp ( \pm \xi) s^{\gamma / 2-1 / 4} \sum_{p=0}^{\infty}(-1)^{p} c_{p}(\gamma) \xi^{-p}$, as $|\mathrm{s}| \rightarrow \infty$

with $\xi=(2 / 3) s^{3 / 2}$ and

$$
c_{0}=1 ; c_{p}(\gamma)=\sum_{l=0}^{2 p} \frac{(-1)^{l} 2^{p} \Gamma(p+l+1 / 2) \Gamma(\gamma+1)}{\sqrt{\pi} 3^{l+p} l !(2 p-l) ! \Gamma(\gamma+1-2 p+l)}, \quad p \geq 0 .
$$

In Figure 2, level contours in the complex $y$ plane of $\Re e\left(\psi_{\theta}\right)$ and $\Im m\left(\psi_{\theta}\right)$ for $\theta=\pi / 2$ are shown. Only the level contours going through the saddle points and $y=0$ are plotted. Also drawn on this figure are the deformed contours $\mathcal{D}_{1}$ and $\mathcal{C}_{1}$. It shows that the contour $\mathcal{D}_{1}$ goes only through $y_{s}^{-}$while $\mathcal{C}_{1}$ goes though $y_{s}^{+}$and encircles 0 . This implies that when $|s| \rightarrow \infty$ with $\arg (s)=$ $\pi / 2$, the only contribution to $A_{1}(s, \gamma)$ is proportional to $\bar{p}^{-}$, while $B_{1}(s, \gamma)$ has a contribution proportional to $\bar{p}^{+}$plus a contribution from 0 .

By analyzing the level contours of $\psi$ for other values of $\theta$, we can determine the contributions to the asymptotic expansions of $A_{1}$ and $B_{1}$ for all values of $\arg (s)$ in the interval $[0,2 \pi]$. The results can be summarized as follows: For $0 \leq \arg (s)<2 \pi / 3$,

$$
\begin{aligned}
& A_{1}(s, \gamma) \sim e^{i \pi(\gamma-1 / 2)} \bar{p}^{-}(s, \gamma) \\
& B_{1}(s, \gamma) \sim \bar{p}^{(0)}(s, \gamma)+\left(e^{2 i \pi \gamma}-1\right) \bar{p}^{+}(s, \gamma) .
\end{aligned}
$$

For $2 \pi / 3<\arg (s)<4 \pi / 3$, 


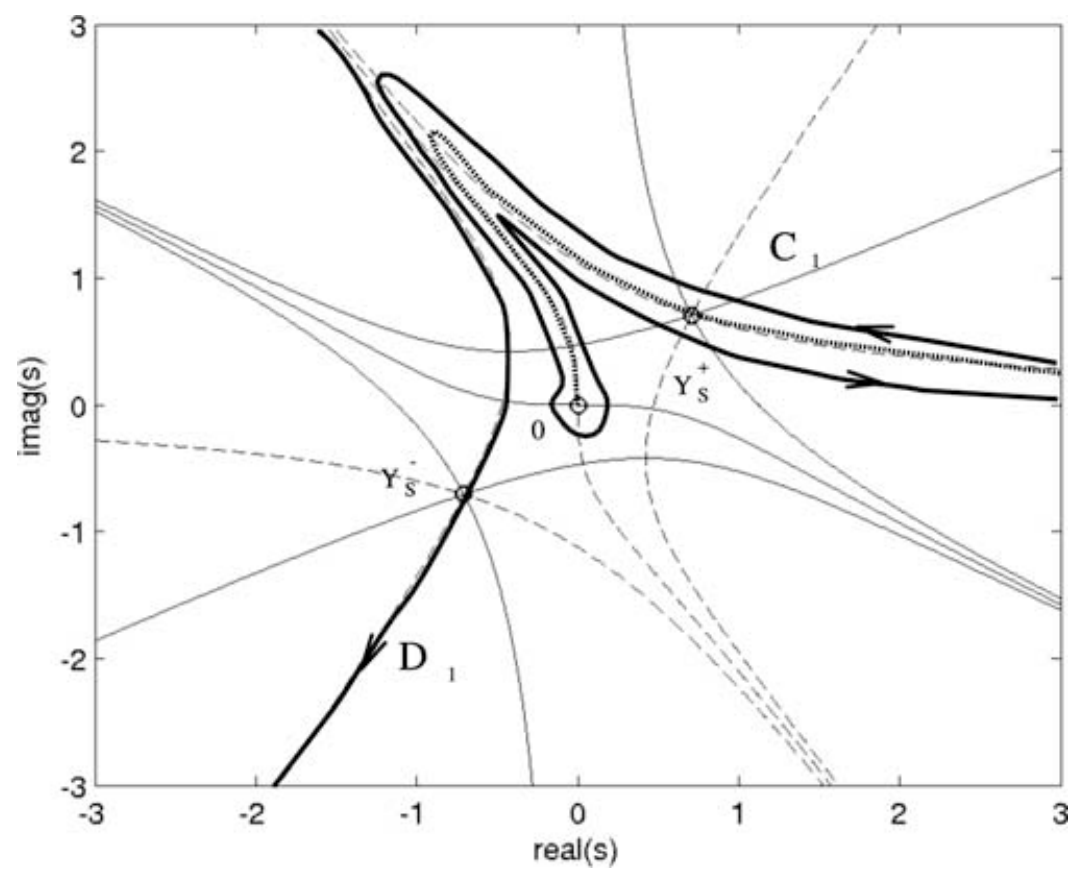

Figure 2. Illustration of the deformation of the contours $\mathcal{C}_{1}$ and $\mathcal{D}_{1}$ in the complex z-plane for $\theta=\pi / 2$. The branch cut is indicated by the dotted line. Thin solid lines and thin dashed lines are constant levels of $\Re e\left(\psi_{\theta}\right)$ and of $\Im m\left(\psi_{\theta}\right)$, respectively.

$$
\begin{aligned}
& A_{1}(s, \gamma) \sim e^{i \pi(\gamma-1 / 2)} \bar{p}^{-}(s, \gamma)+\bar{p}^{(0)}(s, \gamma)+\bar{p}^{+}(s, \gamma) \\
& B_{1}(s, \gamma) \sim \bar{p}^{(0)}(s, \gamma) .
\end{aligned}
$$

For $4 \pi / 3<\arg (s)<2 \pi$,

$$
\begin{aligned}
& A_{1}(s, \gamma) \sim \bar{p}^{+}(s, \gamma) \\
& B_{1}(s, \gamma) \sim \bar{p}^{(0)}(s, \gamma)-2 \sin (\pi \gamma) \bar{p}^{-}(s, \gamma) .
\end{aligned}
$$

Note that for $0<\arg (s)<\pi / 3$ and $\pi<\arg (s)<5 \pi / 3, \bar{p}^{+}(s, \gamma)$ is dominant and $\bar{p}^{-}(s, \gamma)$ is recessive while for $\pi / 3<\arg (s)<\pi$ and $5 \pi / 3<$ $\arg (s)<2 \pi, p^{-}(s, \gamma)$ is dominant and $\bar{p}^{+}(s, \gamma)$ is recessive. The function $A_{1}(s, \gamma)$ is therefore recessive for $0<\arg (s)<\pi / 3$ and $5 \pi / 3<\arg (s)<2 \pi$, and dominant for $\pi / 3<\arg (s)<5 \pi / 3$. The function $B_{1}(s, \gamma)$ has a balanced behavior in the angular sector $\pi / 3<\arg (s)<5 \pi / 3$ and is dominant for $0<$ $\arg (s)<\pi / 3$ and $5 \pi / 3<\arg (s)<2 \pi$. From an asymptotic point of view (in the sense of Poincaré), one obtains from the above expressions (as $|s| \rightarrow \infty)$ :

$$
\begin{aligned}
A_{1}(s, \gamma) \sim e^{i \pi(\gamma-1 / 2)} \bar{p}^{-}(s, \gamma), & 0 \leq \arg (s)<\pi \\
A_{1}(s, \gamma) \sim \bar{p}^{+}(s, \gamma), & \pi<\arg (s)<2 \pi
\end{aligned}
$$




$$
\begin{aligned}
& A_{1}(s, \gamma) \sim e^{i \pi(\gamma-1 / 2)} \bar{p}^{-}(s, \gamma) \\
& \quad+\bar{p}^{(0)}(s, \gamma)+\bar{p}^{+}(s, \gamma), \quad \arg (s)=\pi
\end{aligned}
$$

and

$$
\begin{aligned}
B_{1}(s, \gamma) \sim\left(e^{2 i \pi \gamma}-1\right) \bar{p}^{+}(s, \gamma), & 0 \leq \arg (s)<\pi / 3 \\
B_{1}(s, \gamma) \sim \bar{p}^{(0)}(s, \gamma), & \pi / 3<\arg (s)<5 \pi / 3 \\
B_{1}(s, \gamma) \sim-2 \sin (\pi \gamma) \bar{p}^{-}(s, \gamma), & 5 \pi / 3<\arg (s)<2 \pi \\
B_{1}(s, \gamma) \sim \bar{p}^{(0)}(s, \gamma)+\left(e^{2 i \pi \gamma}-1\right) \bar{p}^{+}(s, \gamma), & \arg (s)=\pi / 3 \\
B_{1}(s, \gamma) \sim \bar{p}^{(0)}(s, \gamma)-2 \sin (\pi \gamma) \bar{p}^{-}(s, \gamma), & \arg (s)=5 \pi / 3 .
\end{aligned}
$$

From the above expressions, one can check that the asymptotic behavior of the solutions $A_{1}$ and $B_{1}$ is uniform in the (Stokes) sector $S_{k}$ bounded by the (Stokes) lines $L_{k}=\{s, \arg (s)=(2+k) \pi / 3, k=1,2,3\}$ displayed in Figure 3. The apparent discontinuity in the above expressions in the sector $S_{1}$ is due to the choice of definition for $\arg (s)$. One can easily show that (40a) and (41a) are actually valid in $S_{1}$ defined as $-\pi / 3<\arg (s)<\pi / 3$. Similarly, (40b) and (41c) are valid in $S_{1}$ defined as $5 \pi / 3<\arg (s)<7 \pi / 3$. The behavior of the other solutions $A_{2}, A_{3}, B_{2}$, and $B_{3}$ can be obtained from the rotation formulas (29) and (30). In the next section, we show that the critical-layer solutions $A_{1}$ and $B_{1}$ match the inviscid and viscous approximations obtained in Sections 2.2 and 2.3.

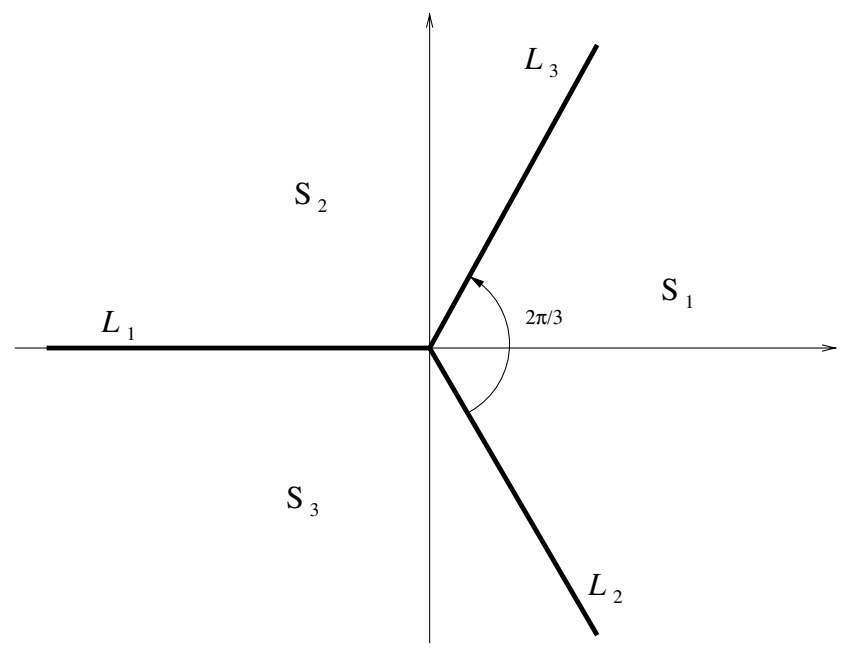

Figure 3. Definition of the Stokes sectors $S_{k}$ and Stokes lines $L_{k}$ in the complex $s$-plane. 


\section{Matching of critical layer solutions to outer solutions}

In this section, the leading order matching between the critical layer solutions and the outer viscous and inviscid approximations obtained in section 2 is performed. For this purpose, it is first useful to write the leading-order term of each critical-layer contribution $\left(\bar{p}^{(0)}, \bar{p}^{-}\right.$, and $\left.\bar{p}^{+}\right)$as a function of the outer variable. Using $s=\left(\Phi_{c}^{\prime}\right)^{1 / 3} \operatorname{Re}^{1 / 3}\left(r-r_{c}\right)$, one obtains at leading order from (34) and (35),

$$
\bar{p}^{(0)} \sim \frac{\left(\Phi_{c}^{\prime} R e\right)^{-(\gamma+1) / 3 e^{2 \pi i \gamma}}}{\Gamma(-\gamma)}\left(r-r_{c}\right)^{-(\gamma+1)},
$$

and

$$
\bar{p}^{ \pm} \sim \frac{\left(\Phi_{c}^{\prime} R e\right)^{(2 \gamma-1) / 12}}{2 \sqrt{\pi} i}\left(r-r_{c}\right)^{(\gamma / 2-1 / 4)} \exp \left( \pm(2 / 3)\left(\Phi_{c}^{\prime} R e\right)^{1 / 2}\left(r-r_{c}\right)^{3 / 2}\right),
$$

where $\gamma$ is either of the roots $\gamma^{(a)}$ or $\gamma^{(b)}$ of equation (27).

The relation $s=\left(\Phi_{c}^{\prime}\right)^{1 / 3} R e^{1 / 3}\left(r-r_{c}\right)$ implies that in the complex $r$-plane, the Stokes sectors $S_{k}$ are now issued from $r_{c}$ and have rotated by an angle equal to $\alpha=-\arg \left(\left(\Phi_{c}^{\prime}\right)^{1 / 3}\right)$. For instance, for a real critical point of a neutral mode $\left(k\right.$ and $\omega$ real), the rotation angle is $\alpha=-\operatorname{sgn}\left(k W_{0 c}^{\prime}+m \Omega_{0 c}^{\prime}\right) \pi / 6$.

If one defines from $\gamma^{(a)}$ and $\gamma^{(b)}$, the coefficients $\alpha^{(a, b)}=-\gamma^{(a, b)}-1$ and $\beta^{(a, b)}=\gamma^{(a, b)} / 2-1 / 4$, they satisfy (12) and (19), respectively. Thus, $p^{(0)}$ and $p^{ \pm}$are associated with the inviscid and viscous approximations obtained in sections 2.2 and 2.3. More precisely, one has for $\gamma=\gamma^{(a)}$

$$
\bar{p}^{(0)} \sim \frac{\left(\Phi_{c}^{\prime} R e\right)^{-\left(\gamma^{(a)}+1\right) / 3 e^{2 \pi i \gamma(a)}}}{\Gamma\left(-\gamma^{(a)}\right)} p_{0 n v}^{(a)},
$$

and

$$
\bar{p}^{ \pm} \sim \frac{\left(\Phi_{c}^{\prime} R e\right)^{\left(2 \gamma^{(a)}-1\right) / 12}}{2 \sqrt{\pi} i} p_{1 v}^{(a)} \exp \left( \pm(2 / 3)\left(\Phi_{c}^{\prime} R e\right)^{1 / 2}\left(r-r_{c}\right)^{3 / 2}\right) .
$$

Moreover expression (8d) reads, at leading order, near $r_{c}$

$$
p_{n v}^{(a)} \sim p_{0 n v}^{(a)}
$$

and expression (14d), using (15), becomes

$$
p_{v}^{(a) \pm} \sim \frac{p_{1 n v}^{(a)}}{\sqrt{R e}} \exp \left( \pm(2 / 3)\left(\Phi_{c}^{\prime} R e\right)^{1 / 2}\left(r-r_{c}\right)^{3 / 2}\right) .
$$

It follows that the function $B_{1}$ matches the inviscid approximation in the sectors $S_{2}$ and $S_{3}$ and the dominant viscous approximation in the sector $S_{1}$. The function $A_{1}$ matches a solution, which is by contrast always viscous except on the Stokes line $L_{1}$ where it contains a non-viscous contribution. It matches the 
dominant viscous approximation in the sectors $S_{2}$ and $S_{3}$, and the recessive viscous approximation in the sector $S_{1}$.

From the asymptotic expressions (40) and (41) for $A_{1}(s, \gamma)$ and $B_{1}(s, \gamma)$, one can now deduce the form of the outer solutions in the complex neighborhood around $r_{c}$. The outer solution which matches to $B_{1}(s, \gamma)$ is given by

$$
\begin{aligned}
p & \sim C^{+}\left(\gamma^{(a)}\right) \operatorname{Re}^{\gamma^{(a)} / 2+3 / 4} A p_{v}^{(a)+} \\
& +C^{+}\left(\gamma^{(b)}\right) R e^{\gamma^{(b)} / 2+3 / 4} B p_{v}^{(b)+} \quad-\pi / 3<\arg \left(\left(\Phi_{c}^{\prime}\right)^{1 / 3}\left(r-r_{c}\right)\right)<\pi / 3 \\
p & \sim A p_{n v}^{(a)}+B p_{n v}^{(b)} \quad \pi / 3<\arg \left(\left(\Phi_{c}^{\prime}\right)^{1 / 3}\left(r-r_{c}\right)\right)<5 \pi / 3 \\
p & \sim C^{-}\left(\gamma^{(a)}\right) R e^{\gamma^{(a)} / 2+3 / 4} A p_{v}^{(a)-} \\
& +C^{-}\left(\gamma^{(b)}\right) R e^{\gamma^{(b)} / 2+3 / 4} B p_{v}^{(b)-} \quad 5 \pi / 3<\arg \left(\left(\Phi_{c}^{\prime}\right)^{1 / 3}\left(r-r_{c}\right)\right)<7 \pi / 3 \\
p & \sim A\left(p_{n v}^{(a)}+C^{+}\left(\gamma^{(a)}\right) R e^{\gamma^{(a)} / 2+3 / 4} p_{v}^{(a)+}\right) \\
& \left.+B\left(p_{n v}^{(b)}+C^{+}\left(\gamma^{(b)}\right) R e^{\gamma^{(b)} / 2+3 / 4} p_{v}^{(b)+}\right)\right) \\
p & \sim A\left(p_{n v}^{(a)}+C^{-}\left(\gamma^{(a)}\right) R e^{\gamma^{(a)} / 2+3 / 4} p_{v}^{(a)-}\right) \\
& \left.+B\left(p_{n v}^{(b)}+C^{-}\left(\gamma^{(b)}\right) R e^{\gamma^{(b)} / 2+3 / 4} p_{v}^{(b)-}\right)\right) \quad \arg \left(\left(\Phi_{c}^{\prime}\right)^{1 / 3}\left(r-r_{c}\right)\right)=\pi / 3
\end{aligned}
$$

where $A$ and $B$ are two independent constants and

$$
\begin{gathered}
C^{+}(\gamma)=\frac{1-\exp (-2 \pi i \gamma)}{2 i \sqrt{\pi}}\left(\Phi_{c}^{\prime}\right)^{\gamma / 2+1 / 4} \Gamma(-\gamma), \\
C^{-}(\gamma)=\frac{i \sin (\pi \gamma)}{\sqrt{\pi}} e^{-2 \pi i \gamma}\left(\Phi_{c}^{\prime}\right)^{\gamma / 2+1 / 4} \Gamma(-\gamma) .
\end{gathered}
$$

The outer solution which matches to $A_{1}(s, \gamma)$ reads

$$
\begin{aligned}
& p \sim A p_{v}^{(a)-}+B p_{v}^{(b)-} \quad-\pi<\arg \left(\left(\Phi_{c}^{\prime}\right)^{1 / 3}\left(r-r_{c}\right)\right)<\pi \\
& p \sim D^{+}\left(\gamma^{(a)}\right) A p_{v}^{(a)+}+D^{+}\left(\gamma^{(b)}\right) B p_{v}^{(b)+} \quad \pi<\arg \left(\left(\Phi_{c}^{\prime}\right)^{1 / 3}\left(r-r_{c}\right)\right)<3 \pi \\
& p \sim A\left(p_{v}^{(a)-}+D^{+}\left(\gamma^{(a)}\right) p_{v}^{(a)+}+D\left(\gamma^{(a)}\right) R e^{-\gamma^{(a)} / 2-3 / 4} p_{n v}^{(a)}\right) \\
& +B\left(p_{v}^{(b)-}+D^{+}\left(\gamma^{(b)}\right) p_{v}^{(b)+}+D\left(\gamma^{(b)}\right) R e^{-\gamma^{(b)} / 2-3 / 4} p_{n v}^{(b)}\right) \\
& \arg \left(\left(\Phi_{c}^{\prime}\right)^{1 / 3}\left(r-r_{c}\right)\right)=\pi
\end{aligned}
$$

where

$$
\begin{aligned}
D^{+}(\gamma) & =i \exp (-i \pi \gamma) \\
D(\gamma) & =-\frac{2 \exp (i \pi \gamma) \sqrt{\pi}\left(\Phi_{c}^{\prime}\right)^{-\gamma / 2-1 / 4}}{\Gamma(-\gamma)} .
\end{aligned}
$$


Note that we have extended the domain of uniform approximation of the function $A_{1}(s, \gamma)$ from $-\pi / 3<\arg (s)<\pi$ to $-\pi<\arg (s)<\pi$ and from $\pi<\arg (s)<7 \pi / 3$ to $\pi<\arg (s)<3 \pi$. The other critical-layer solutions $A_{2}, A_{3}, B_{2}$, and $B_{3}$ provide similar expressions for the outer solutions. The result is just obtained by taking a different definition for the cubic root in $s=$ $\left(\Phi_{c}^{\prime}\right)^{1 / 3}\left(r-r_{c}\right)$.

\section{Conclusion}

In this paper, we have resolved the (simple) singularities that inviscid approximations and viscous approximations for three-dimensional normal modes exhibit at the radial coordinates $r_{c}$, where $\omega=m \Omega_{0}+k W_{0}$. These singularities have been smoothed out in a viscous critical layer of width $O\left(R e^{-1 / 3}\right)$. The critical layer equation has been shown to be the same as for planar stratified shear flows with a unit Prandtl number. Integral representations of two typical critical-layer solutions have been derived and used to deduce the behavior of the normal mode amplitude in the complex plane around $r_{c}$.

We have proved that one of these solutions $\left(B_{1}\right)$ can be matched to an inviscid approximation in a sector around the critical point whose angular range is $4 \pi / 3$. This means that there exist solutions which are non-viscous on either side of the singularity. Moreover, the uniform validity in this $4 \pi / 3$ sector guarantees that the Frobenius expansions of such a solution on both sides of $r_{c}$ differ by a simple $\pi$ phase jump only, as is the case for planar shear flows. In the third $2 \pi / 3$ sector around $r_{c}$, the critical-layer solution is dominant: it matches a dominant viscous solution as $r-r_{c}$ increases. An asymptotic expression of the solution in this sector has been obtained.

The solution associated with $B_{1}$ is expected to describe damped inviscid normal modes. Such modes are known to exist for a Gaussian vortex without axial flow [13, 22]. Moreover, if they are not too damped, they can appear spontaneously by a resonance mechanism via the so-called elliptical instability $[13,23]$. The present analysis provides the spatial structure of these modes.

The second type of critical layer solution $\left(A_{1}\right)$ has been shown to have a recessive viscous behavior in a $2 \pi / 3$ angular sector and a dominant viscous behavior elsewhere. Explicit asymptotic expressions for the solution around $r_{c}$ have been provided and matched to dominant and recessive viscous WKBJ approximations. This type of solution is expected to be present in the asymptotic structure of viscous modes. In particular, as for planar shear flows, it will appear in the structure of boundary layer modes.

We have also shown how other solutions can be obtained by simple rotation formulas. Together, they form a complete set of solutions, which can be used to represent any linear normal mode eigenfunction around a critical point. 


\section{Acknowledgment}

I am grateful to S. Maslowe for having pointed out the resemblance of the present work with critical layer analysis in stratified shear flows. I would also like to thank S. Maslowe and D. Fabre for their comments on an early version of this paper.

\section{References}

1. C. C. Lin, The Theory of Hydrodynamics Stability, Cambridge University Press, 1955.

2. P. G. Drazin and W. H. ReID, Hydrodynamic Stability, Cambridge University Press, 1981.

3. P. J. Schmid and D. S. Henningson, Stability and Transition in Shear Flows in Applied Mathematical Sciences, Vol. 142, Springer, 2001.

4. C. C. Lin, On the stability of two-dimensional parallel flows, Quart. Appl. Math. 3:117-42, 218-34, and 277-301 (1945).

5. D. Koppel, On the stability of flow of a thermally stratified fluid under the action of gravity, J. Math. Phys. 5:963-982 (1964).

6. P. BALDwIN and P. H. RoBerts, The critical layer in stratified shear flow, Mathematika 17:102-119 (1970).

7. S. A. Maslowe, Critical layers in shear flows, Ann. Rev. Fluid Mech. 18:405-432, 1986.

8. S. J. Cowley and X. Wu, Asymptotic Approaches to Transition Modelling, Agard Report 793:3.1-3.38 (1994).

9. S. M. Churilov and I. G. Shukhman, The nonlinear critical layer resulting from the spatial or temporal evolution of weakly unstable disturbances in shear flows, J. Fluid Mech. 318:189-221 (1996).

10. R. L. Ash and M. R. Khorrami, Vortex stability, in Fluid Vortices (S. I. Green, Ed.), pp. 317-372. Kluwer Academic Publishers, 1995.

11. M. Lessen, P. J. Singh, and F. Paillet, The stability of a trailing line vortex. Part 1. Inviscid theory, J. Fluid Mech. 63:753-763 (1974).

12. C. Olendraru, A. Sellier, M. Rossi, and P. Huerre, Inviscid instability of the Batchelor vortex: Absolute/convective transition and spatial branches, Phys. Fluids 11:1805-1820, (1999).

13. D. SIPP and L. JACQUIN, Widnall instabilities in vortex pairs, Phys. Fluids 15:1861-1874, 2003.

14. T. H. Hughes and W. H. ReID, The stability of spiral flow between rotating cylinders, Phil. Trans. Roy. Soc. 263A:57-91 (1968).

15. K. S. GAGE and W. H. ReID, The stability of thermally stratified plane Poiseuille flow, $J$. Fluid. Mech. 33:21-32 (1968).

16. K. S. GAGE, The effect of stable thermal stratification on the stability of viscous parallel flows, J. Fluid Mech. 47:1-20, 1971.

17. W. H. ReID, Composite approximations to the Orr-Sommerfeld equation, Stud. Appl. Math. 51:341-68, 1972.

18. P. G. Saffman, Vortex Dynamics, Cambridge University Press, 1992.

19. W. Wasow, Linear Turning Point Theory, Springer-Verlag, 1985.

20. A. ERDÉLYI, Asymptotic Expansions, Dover Publications, 1956. 
21. R. Wong, Asymptotic Approximations of Integrals, Academic Press, 1989.

22. D. Fabre, Ph.D. Thesis, ONERA/Université Paris VI, 2002.

23. S. Le DizÈs and F. LAPORTE, Theoretical predictions for the elliptic instability in a two-vortex flow, J. Fluid Mech. 471:169-201, 2002.

\section{Universités D’AiX-MARSEILLE I \& II}

(Received May 9, 2003) 